\title{
Magnetization switching driven by current-induced torque from weakly spin-orbit coupled $\mathrm{Zr}$
}

\author{
Z. C. Zheng, ${ }^{1, *}$ Q. X. Guo,,${ }^{1,2,{ }^{*}}$ D. Jo, ${ }^{3}$ D. Go $\odot,{ }^{3, \dagger}$ L. H. Wang, ${ }^{4}$ H. C. Chen,,${ }^{5}$ W. Yin,${ }^{5}$ X. M. Wang, ${ }^{6}$ \\ G. H. Yu, ${ }^{1}$ W. He, ${ }^{2}$ H.-W. Lee, ${ }^{3}$ J. Teng, ${ }^{1, *}$ and T. Zhu ${ }^{2,5,7, \S}$ \\ ${ }^{1}$ Department of Materials Physics and Chemistry, University of Science and Technology Beijing, Beijing 100083, China \\ ${ }^{2}$ Beijing National Laboratory for Condensed Matter Physics and Institute of Physics, Chinese Academy of Sciences, Beijing 100190, China \\ ${ }^{3}$ Department of Physics, Pohang University of Science and Technology, Pohang 37673, Korea \\ ${ }^{4}$ Institute of Microstructure and Property of Advanced Materials, Beijing Key Laboratory of Microstructure \\ and Property of Advanced Materials, Beijing University of Technology, Beijing 100124, China \\ ${ }^{5}$ Spallation Neutron Source Science Center, Dongguan 523803, China \\ ${ }^{6}$ Collaborative Innovation Center of Advanced Steel Technology, University of Science and Technology Beijing, Beijing 100083, China \\ ${ }^{7}$ Songshan Lake Materials Laboratory, Dongguan 523808, China
}

(Received 31 October 2019; revised manuscript received 19 December 2019; accepted 20 December 2019; published 5 February 2020)

\begin{abstract}
Current-induced magnetization switching (CIMS) via the spin-orbit torque is technologically important for applications of spintronic devices. Existing experimental studies are focused on $5 d$ heavy metals with large spinorbit coupling but theoretical studies indicate that $3 d$ or $4 d$ light metals with weak spin-orbit coupling may also generate sizable torque through the strong orbital Hall effect. However, CIMS has seldom been experimentally demonstrated using $3 d$ or $4 d$ light metals. Here, we report sizable current-induced torque and a robust CIMS in weakly spin-orbit coupled $\mathrm{Zr}$ based perpendicular magnetized multilayers. Current-induced effective torque field changes its sign as the $\mathrm{Zr}$ layer thickness varies, indicating the competition of multiple effects. Possible roles of the orbital Hall effect in the competition are discussed. Our findings widen the material scope of the CIMS and imply possible applications of the orbital Hall effect.
\end{abstract}

DOI: 10.1103/PhysRevResearch.2.013127

\section{INTRODUCTION}

The manipulation of the magnetization in a magnetic multilayer can be achieved in an energy-efficient and fast way by current-induced magnetization switching (CIMS) [1-3] in a $5 d$ heavy metal buffered $\mathrm{CoFeB} / \mathrm{MgO}$ multilayer [4-10], due to the spin angular momentum transfer from the heavy metal to $\mathrm{CoFeB}$ by the large spin Hall effect (SHE) in these $5 d$ heavy metals, such as Pt [11], Ta [3], and W [12]. As for $3 d$ or $4 d$ light metals with weak spin-orbit coupling (SOC), few CIMS experiments have been reported [13,14]. However, theories predict that the orbital Hall effect (OHE), a different version of the angular momentum generation effect, can be large in the transition metals despite the weak SOC [15-18]. The calculations show that their orbital Hall conductivities (OHCs) are about an order of magnitude larger than the spin Hall conductivities (SHCs) $[15,16]$. In particular, a re-

\footnotetext{
${ }^{*}$ These authors contributed equally to this work.

${ }^{\dagger}$ Also works at Basic Science Research Institute, Pohang University of Science and Technology, Pohang 37673, Korea and Peter Grünberg Institut and Institute for Advanced Simulation, Forschungszentrum Jülich and JARA, 52428 Jülich, Germany.

‡Corresponding author: tengjiao@mater.ustb.edu.cn

${ }^{\S}$ Corresponding author: tzhu@iphy.ac.cn

Published by the American Physical Society under the terms of the Creative Commons Attribution 4.0 International license. Further distribution of this work must maintain attribution to the author(s) and the published article's title, journal citation, and DOI.
}

cent calculation reports that intrinsic $\mathrm{OHC}$ can be gigantic, $\sigma_{\mathrm{OH}} \sim 10^{4}(\hbar / 2|e|)(\Omega \mathrm{cm})^{-1}$, in some materials with negligible SOC [18].

In the presence of SHE, a charge current converts into a spin current due to the spin-orbit interaction, which generates a spin-orbit torque (SOT) in the adjacent ferromagnet (FM) layer. In the presence of OHE, a charge current can be converted to a flow carrying orbital angular momentum, which is defined as orbital current. The orbital current may be either transformed to the spin current within a nonmagnetic metal (thus providing a mechanism to generate SHE of the nonmagnetic metal), or may be injected to an adjacent FM layer. According to a recent theoretical calculation [19], the injection of the orbital current generates an orbital torque (OT) in the FM layer. Since OT has similar properties to SOT, OT should also achieve CIMS in a perpendicularly magnetized multilayer. In this respect, a good test system to experimentally study OT and OHE is a multilayer consisting of a light metal (LM) and a FM with the inversion symmetry broken along the film growth direction. Thus, the search for a $\mathrm{LM} / \mathrm{FM} / \mathrm{MgO}$ multilayer with clear CIMS behavior is valuable not only for widening the material scope of CIMS but also for studying OT and OHE.

Here we demonstrate the CIMS and the measurement of torque in the perpendicularly magnetized $\mathrm{Zr} / \mathrm{CoFeB} / \mathrm{MgO}$ multilayers. $\mathrm{Zr}$ is a $4 d$ light metal. Our theoretical calculation shows that the $\mathrm{OHC}$ of $\mathrm{Zr}$ is more than an order of magnitude larger than its SHC. Similar to the SOT, OT-induced effective fields in the $\mathrm{Zr} / \mathrm{CoFeB} / \mathrm{MgO}$ multilayer also have two components, $H_{L}$ and $H_{T}$, namely, longitudinal and transverse 

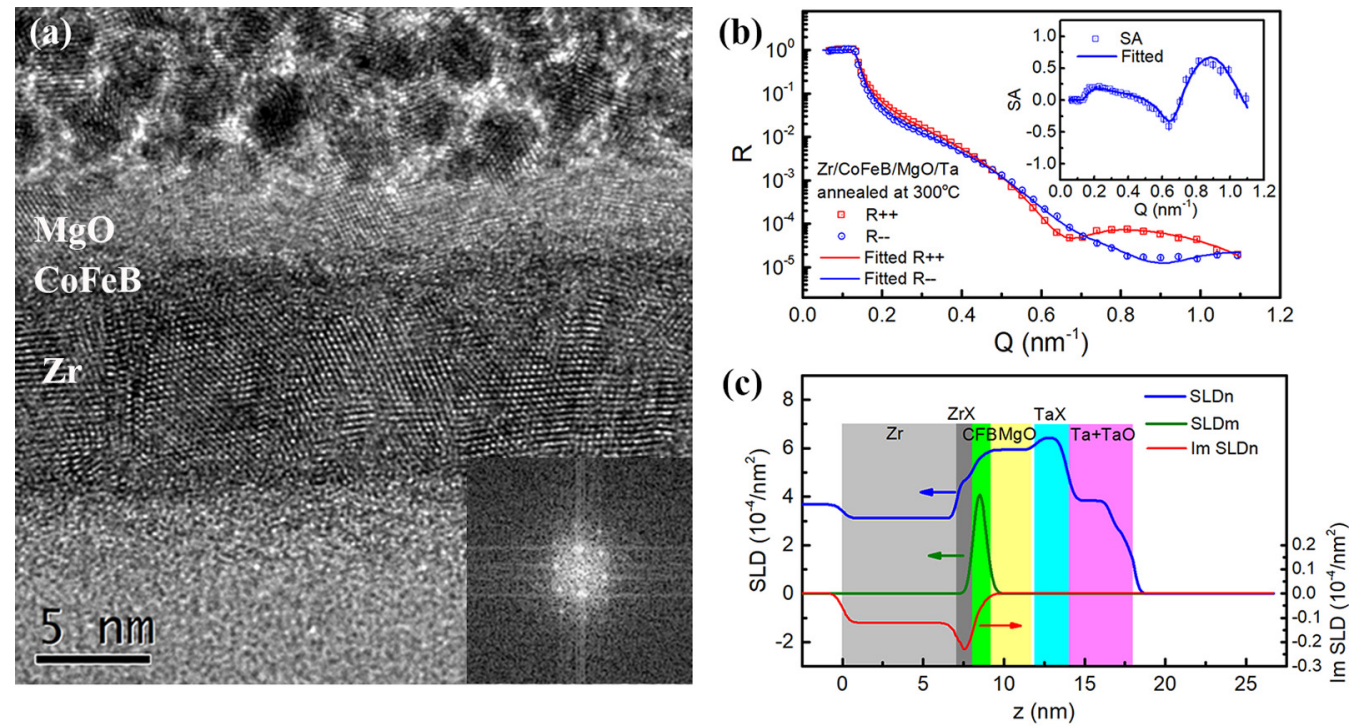

FIG. 1. Structure of $\mathrm{Zr} / \mathrm{CoFeB} / \mathrm{MgO}$ annealed at $300{ }^{\circ} \mathrm{C}$. (a) High-resolution TEM image of $\mathrm{Zr}(8) / \mathrm{CoFeB}(1.1) / \mathrm{MgO}(3)$ sample annealed at $300^{\circ} \mathrm{C}$. The inset shows the fast Fourier transform pattern from the $\mathrm{Zr}$ layer, indicating hep structure. (b) $\mathrm{PNR}$ curves of the $\mathrm{Zr} / \mathrm{CoFeB} / \mathrm{MgO} / \mathrm{Ta}$ multilayer measured at the applied magnetic field $H=9000$ Oe at room temperature. Here, the 3-nm-thickness Ta layer is a capping layer to protect the $\mathrm{Zr} / \mathrm{CoFeB} / \mathrm{MgO}$ film. The inset shows the related spin asymmetry (SA) curve. Symbols correspond to the experimental data points, while the solid lines show the fitted curves. (c) The fitted nuclear and magnetic SLD ( $\operatorname{SLD}_{n}$ and $\mathrm{SLD}_{m}$ ) profiles.

configurations. Thus torque measurement methods will measure the sum of SOT and OT. Based on the harmonic Hall voltage method in a patterned Hall bar, a sign change of the effective torque fields with the increasing $\mathrm{Zr}$ thickness has been observed. The sign reversal behavior of the torque effective fields was also confirmed by CIMS measurements, suggesting possible competitions between SOT, OT, and also torque due to the interfacial Rashba effect in the $\mathrm{Zr} / \mathrm{CoFeB} / \mathrm{MgO}$ multilayers.

\section{EXPERIMENTS}

Multilayers films with a structure of $\mathrm{Zr}\left(t_{\mathrm{Zr}}\right) / \mathrm{Co}_{40} \mathrm{Fe}_{40} \mathrm{~B}_{20}$ (1.1) $/ \mathrm{MgO}(3)$ (numbers in parentheses are thicknesses in nanometers) were deposited on thermally oxidized $\mathrm{Si} / \mathrm{SiO}_{2}$ substrates at room temperature by a magnetron sputtering system. The thickness of the $\mathrm{Zr}$ layer was systematically varied from $2.2 \mathrm{~nm}$ to $16.2 \mathrm{~nm}$. The base pressure of the sputtering system was less than $8 \times 10^{-6} \mathrm{~Pa}$. The $\mathrm{Zr}$ and $\mathrm{Co}_{40} \mathrm{Fe}_{40} \mathrm{~B}_{20}(\mathrm{CoFeB})$ layers were deposited by using a direct current (DC) power source and the $\mathrm{MgO}$ layer was deposited by using a radio-frequency power source. The deposition rates for $\mathrm{Zr}, \mathrm{CoFeB}$, and $\mathrm{MgO}$ layers were $0.54 \AA / \mathrm{s}, 0.68 \AA / \mathrm{s}$, and $0.1 \AA / \mathrm{s}$, respectively. The films were annealed in a vacuum, which was less than $1 \times 10^{-5} \mathrm{~Pa}$, at $300^{\circ} \mathrm{C}$ for $1 \mathrm{~h}$ to obtain perpendicular magnetic anisotropy. The $\mathrm{Zr} / \mathrm{CoFeB} / \mathrm{MgO}$ films were patterned into Hall bars of width $10 \mu \mathrm{m}$ by standard photolithography combined with an argon ion etching technique. The anomalous Hall effect (AHE) and CIMS were measured by using a room-temperature multipurpose transport measurement system with a $1 \mathrm{~T}$ electromagnet, a Keithley 2182A, and a Keithley 6221 was employed as the source for the DC and pulse measurements. The transmission electron microscopy (TEM) analysis was performed in a FEI Tecnai G2 F20 microscope, operated at $200 \mathrm{kV}$. The samples for TEM analysis were prepared by the focus ion beam with Helios Nanolab
600. Figure 1(a) presents a high-resolution TEM image of the cross-sectional $\mathrm{Zr}(8.1) / \mathrm{CoFeB}(1.1) / \mathrm{MgO}(3)$ multilayer after annealing at $300^{\circ} \mathrm{C}$. The $\mathrm{CoFeB}$ layer is nearly amorphous, while the $\mathrm{MgO}$ layer exhibits a polycrystalline structure. The $\mathrm{Zr}$ underlayer is also polycrystalline. The inset is the fast Fourier transform pattern, indicating a clear hexagonal-closepacked (hcp) structure of the $\mathrm{Zr}$ layer.

To further understand the layer structure of the annealed $\mathrm{Zr} / \mathrm{CoFeB} / \mathrm{MgO}$ multilayer, the room-temperature polarized neutron reflectometry (PNR) measurement was carried out on the Multipurpose Reflectometer at the China Spallation Neutron Source, which is a unique depth-resolved technique to understand the magnetic properties in a magnetic layer or multilayer due to the high penetration depth of neutrons [20]. The neutron reflectivity curves are recorded at room temperature as the function of momentum transfer $Q=4 \pi \sin \theta / \lambda$, where $\lambda$ is the neutron wavelength and $\theta$ is the incident angle between the neutron beam and the film plane. To access a broad momentum transfer range, reflected neutrons were collected at different incident angles. Figure 1(b) shows the non-spin-flip reflectivity $R^{++}$and $R^{--}$curves measured under the applied field of 9000 Oe. Non-spin-flip reflectivities indicate that the spin polarizations are the same for the incoming and reflected neutrons. The spin asymmetry (SA) is defined as $\mathrm{SA}=\left(R^{++}-R^{--}\right) /\left(R^{++}+R^{--}\right)$, which is very sensitive to the magnetization of the film. The obtained $R^{++}$and $R^{--}$ curves were simulated using the SimuReflec program [21]. From the simulation, the depth dependences of nuclear and magnetic scattering length densities $\left(\mathrm{SLD}_{n}\right.$ and $\left.\mathrm{SLD}_{m}\right)$ were derived as a function of film thickness as shown in Fig. 1(c). A reasonable fitting was obtained with a multilayer model for the annealed $\mathrm{Zr} / \mathrm{CoFeB} / \mathrm{MgO}$ sample, containing the interface between the $\mathrm{Zr}$ and $\mathrm{CoFeB}$ layers and fixing the nuclear scattering length density of the $\mathrm{Zr}$ and $\mathrm{MgO}$ layers with their bulk values. Due to the noticeable neutron absorption of $\mathrm{B}$ nuclei, the imaginary part of $\mathrm{SLD}_{n}$ is also considered during 


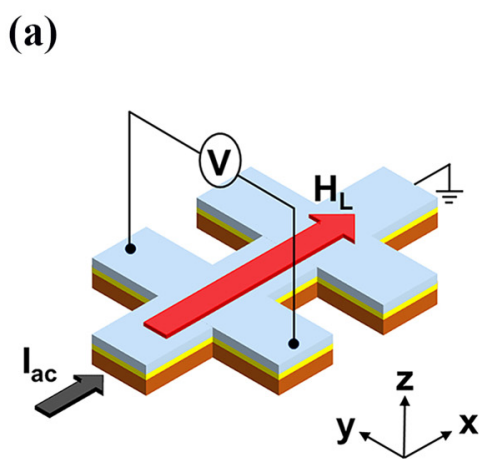

(b)

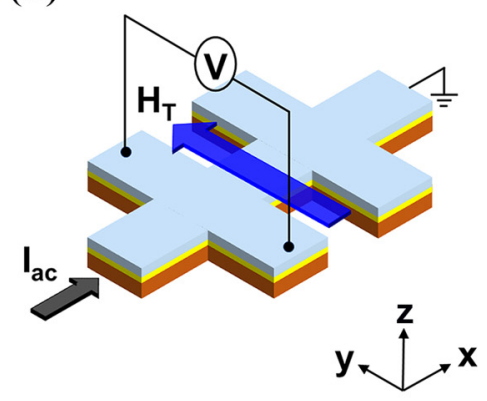

(c)

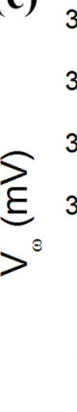

(e)

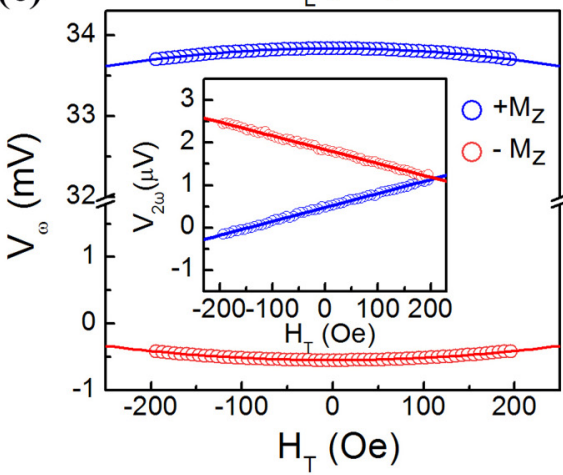

(d)

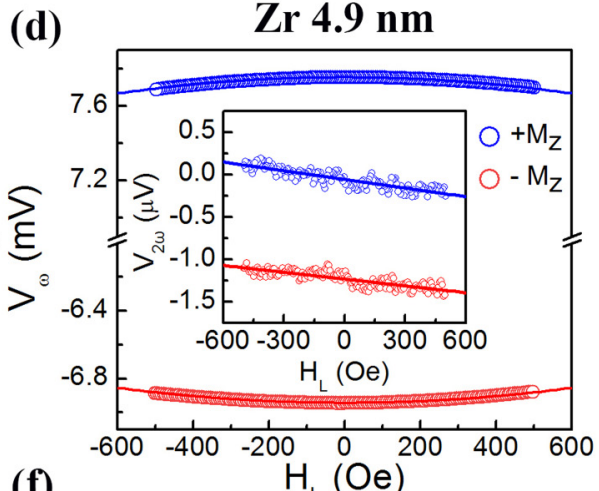

(f)

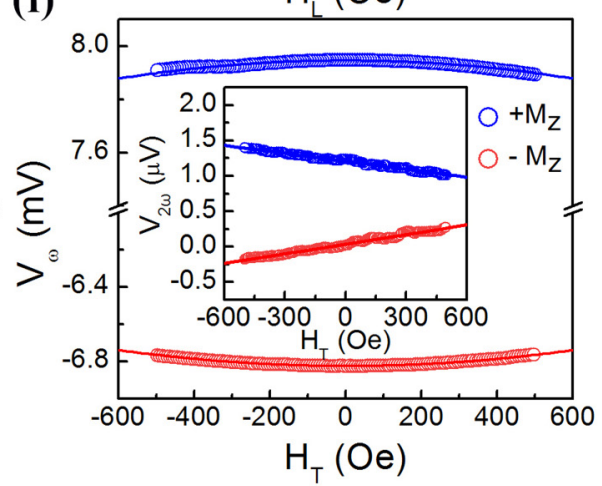

FIG. 2. Schematics of harmonic measurement and current-induced torque effective fields. (a), (b) Longitudinal and transverse orbitaltorque-induced effective field measurements. The first-harmonic voltages $V_{\omega}$ versus in-plane longitudinal $H_{L}$ [(c) $\mathrm{Zr} 2.2 \mathrm{~nm}$ and (d) $\left.\mathrm{Zr} 4.9 \mathrm{~nm}\right]$ and transverse $H_{T}$ [(e) $\mathrm{Zr} 2.2 \mathrm{~nm}$ and (f) $\mathrm{Zr} 4.9 \mathrm{~nm}$ ] swept magnetic field. The inset of each figure corresponds to the second-harmonic voltages $V_{2 \omega}$ as a function of $H_{L}$ [(c) and (d)] and $H_{T}$ [(e) and (f)]. The blue and red symbols correspond to $+M_{z}$ and $-M_{z}$ states, respectively. Solid lines represent the quadratic and linear fittings for $V_{\omega}$ and $V_{2 \omega}$, respectively.

the fitting. As shown in our fitted model, a high region of the imaginary part of $\mathrm{SLD}_{n}\left(\operatorname{ImSLD}_{n}\right)$ can be seen in the interface between $\mathrm{Zr}$ and $\mathrm{CoFeB}$, indicating the diffusion of $\mathrm{B}$ into the $\mathrm{Zr}$ layer. A similar feature can be seen in $\mathrm{Ta} / \mathrm{CoFeB}$ PMA samples by using PNR measurements [22]. The magnetic scattering length density is directly proportional to the saturation magnetization of the sample because $\mathrm{CoFeB}$ should be already saturated by the in-plane magnetic field of 9000 Oe. From the fitting, one obtains $\mathrm{SLD}_{m}=4.06 \times 10^{-4} \mathrm{~nm}^{-2}$. This gives $M_{s}=1400 \mathrm{emu} / \mathrm{cm}^{3}$, which may be due to the $\mathrm{CoFe}$ formation after some of the $\mathrm{B}$ are absorbed by $\mathrm{Zr}$.

\section{RESULTS AND DISCUSSION}

Figures 2(a) and 2(b) show the setup of the harmonic Hall voltage measurement, where a low-frequency sinusoidal current $(133.33 \mathrm{~Hz})$ was passed into the patterned Hall bars and the in-phase first-harmonic and out-of-phase secondharmonic Hall voltages were measured by two Stanford lockin amplifiers. From the harmonic Hall voltage measurements, the current-induced effective torque fields in two configurations, $H_{L}$ and $H_{T}$, namely, longitudinal and transverse configurations, respectively, can be obtained [23,24]. For $H_{L}$ measurement, the magnetic field is swept in the direction of alternating current, while for $H_{T}$ measurement, the magnetic field is swept perpendicular to the current direction. In both measurement configurations, the magnetic field is applied with a small tilting angle (about $2^{\circ}$ ) to the film plane.
Figures 2(c)-2(f) show the first-harmonic Hall voltages $\left(V_{\omega}\right)$ as a function of $H_{L}$ and $H_{T}$ for two samples with $\mathrm{Zr}$ thicknesses of $2.2 \mathrm{~nm}$ and $4.9 \mathrm{~nm}$, respectively. It is clear that the $V_{\omega}$ can be fitted using a parabola dependence. The inset of each figure represents the second-harmonic Hall voltages $\left(V_{2 \omega}\right)$ as a function of $H_{L}$ and $H_{T}$, which shows linear dependence. For the longitudinal field, the slopes of the $V_{2 \omega}$ curves are the same for magnetizations pointing along $+z$ and $-z$, whereas their sign reverses for the transverse field. However, the sign of the slope changes for both longitudinal and transverse fields when the $\mathrm{Zr}$ thickness is increased from $2.2 \mathrm{~nm}$ to $4.9 \mathrm{~nm}$. The polarization of $V_{2 \omega}$ shows that the sign of effective torque is changed when the thickness of $\mathrm{Zr}$ is increased.

The longitudinal effective field $\left(\Delta H_{L}\right)$ and the transverse effective field $\left(\Delta H_{T}\right)$ can be calculated from the $H_{L}$ and $H_{T}$ field dependencies of harmonic voltages, using the following equation when the planar Hall effect is negligible compared to the anomalous Hall effect [25],

$$
\Delta H_{L, T}=-2 \frac{\partial V_{2 \omega} / \partial H_{L, T}}{\partial^{2} V_{\omega} / \partial H_{L, T}^{2}} .
$$

The longitudinal (transverse) torque efficiencies $\left(\beta_{L_{(T)}}\right)$ are defined as $\Delta H_{L(T)} / J_{e}$, to characterize the effective torque fields. The $\mathrm{Zr}$ thickness dependence of the longitudinal effective field per current density $\left(\beta_{L}=\Delta H_{L} / J_{e}\right)$ and the transverse effective field per current density $\left(\beta_{T}=\Delta H_{T} / J_{e}\right)$ are plotted in Figs. 3(a) and 3(b), respectively. Both $\beta_{L}$ and $\beta_{T}$ change their signs when the $\mathrm{Zr}$ thickness is above $3 \mathrm{~nm}$. 


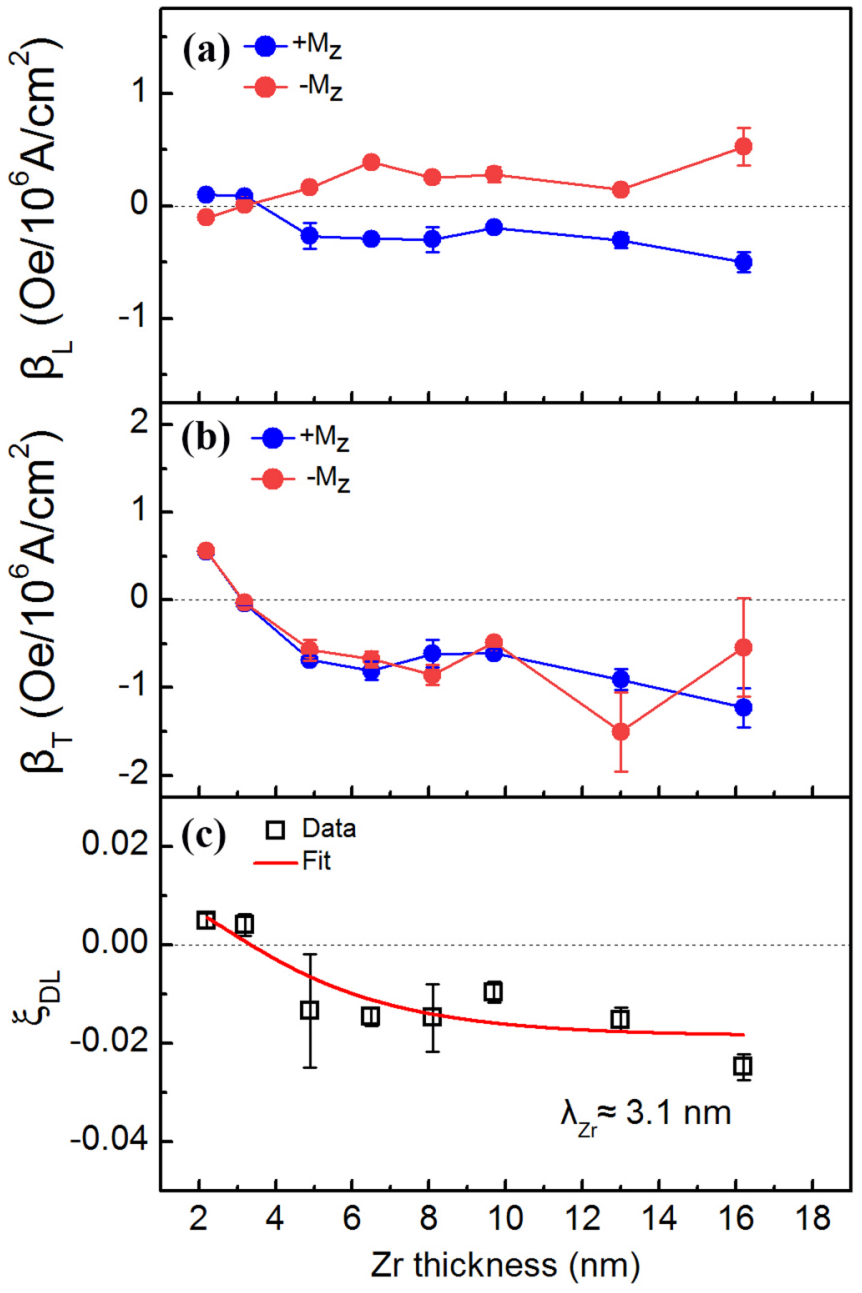

(d)

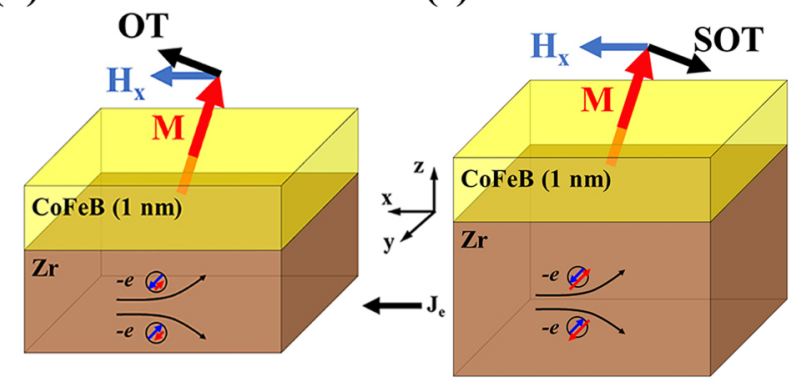

FIG. 3. $\mathrm{Zr}$ thickness dependence of torque efficiency. Longitudinal (a) and transverse (b) torque efficiency, $\beta_{L}=\Delta H_{L} / J_{e}$ and $\beta_{T}=\Delta H_{T} / J_{e}$, respectively, are plotted as a function of $\mathrm{Zr}$ thickness. The effective field is normalized by the current density $\left(J_{e}\right)$ that flows into the $\mathrm{Zr}$ layer. (c) $\mathrm{Zr}$ thickness dependence of the damping-like torque efficiency $\xi_{\text {DL }}$. Illustrations of OT (d) and SOT (e) generated by weakly spin-orbit coupled Zr. In panels (d) and (e), the blue arrows represent the atomic-orbital angular momentum, while the red arrows represent spin angular momentum.

In principle, current-induced torque can generate the fieldlike and damping-like terms, when a spin (or orbital) current polarized along the $\hat{\boldsymbol{y}}$ direction $(\hat{\boldsymbol{\sigma}} \| \hat{\boldsymbol{y}})$ is injected into a FM magnetized along the $\hat{z}$ direction. The damping-like effective torque field can arise through the SHE (or OHE) and the
Rashba effect at the FM/LM interface, which is described as $\vec{\tau}_{\mathrm{DL}} \propto \hat{\mathbf{m}} \times(\hat{\mathbf{y}} \times \hat{\mathbf{m}})$. Here $\hat{\mathbf{m}}$ is the unit vector along the direction of magnetization. Damping-like torque efficiency can be extracted using the following equation:

$$
\xi_{\mathrm{DL}}=2|e| M_{s} t_{\mathrm{CoFeB}} \Delta H_{\mathrm{L}} / \hbar J_{e}=2|e| M_{s} t_{\mathrm{CoFeB}} \beta_{L} / \hbar,
$$

where $e$ is the charge of an electron, $M_{s}$ is the saturation magnetization of $\mathrm{CoFeB}, t_{\mathrm{CoFeB}}$ is the thickness of $\mathrm{CoFeB}$, and $\hbar$ is the reduced Planck constant. Figure 3(c) shows the $t_{\mathrm{Zr}}$ dependence of $\xi_{\mathrm{DL}}$ as determined by using Eq. (2). $\xi_{\mathrm{DL}}$ is positive for the thin $\mathrm{Zr}$ layer and negative for the thick $\mathrm{Zr}$ layer. Current-induced effective torque fields exhibit a significant dependence on the $\mathrm{Zr}$ layer thickness, indicating that there are competing effects, the interfacial Rashba effect and bulk SHE (and possibly OHE). To simplify the description of current-induced torque in a LM with weak SOC, we introduce the effective spin Hall angle $\theta_{\mathrm{SH}}$. If SHE-induced SOT is the dominant source of $\theta_{\mathrm{SH}}, \theta_{\mathrm{SH}}$ represents the ratio of the spin current density $J_{s}$ absorbed by $\mathrm{CoFeB}$ to the charge current density in the $\mathrm{Zr}$ layer, $\theta_{\mathrm{SH}}=J_{s} / J_{c}$. If OHE-induced OT is the dominant source of $\theta_{\mathrm{SH}}, \theta_{\mathrm{SH}}$ is given instead by $J_{o} / J_{c}$, where $J_{o}$ is the effective spin current density in the $\mathrm{Zr}$ layer generated by the orbital current injection into the $\mathrm{Zr}$ layer [19]. When SOT and OT are comparable, $\theta_{\mathrm{SH}}$ should contain both contributions. For simplicity, we assume for now that $\theta_{\mathrm{SH}}$ is dominated by the SHE-induced SOT and consider its competition with the Rasbha-based mechanism of the damping-like torque [26-29]. If we follow the diffusion theory [24,30] and assume that the $\mathrm{Zr} / \mathrm{CoFeB}$ interface has perfect transparency, the damping-like torque efficiency can be expressed as

$$
\xi_{\mathrm{DL}}=\theta_{\mathrm{SH}}\left[1-\operatorname{sech}\left(t_{\mathrm{Zr}} / \lambda_{\mathrm{Zr}}\right)\right]+C,
$$

where $\lambda_{Z r}$ represents the characteristic spin diffusion length of $\mathrm{Zr}$ and $C$ is a constant arising from the interfacial Rashba mechanism. A fit of Eq. (3) to the data [Fig. 3(c)] yields $\theta_{\mathrm{SH}}=$ $-0.03 \pm 0.006, \lambda_{\mathrm{Zr}}=3.1 \pm 1.3 \mathrm{~nm}, C=0.012 \pm 0.01$. According to the Rashba Hamiltonian, the direction of the Rashba-effect-induced spin accumulation is along $\boldsymbol{E} \times \boldsymbol{p}$, where $\boldsymbol{p}$ is the electron momentum. The direction of $\boldsymbol{E}$ for a given interface is only determined by the difference of work function $(\Delta \Phi)$ between two adjacent layers. As for the $\mathrm{Zr} / \mathrm{CoFeB} / \mathrm{MgO}$ multilayer, the work function difference is expected to be $-0.75 \mathrm{eV}$ between the $\mathrm{Zr}\left(\Phi_{\mathrm{Zr}}=4.05 \mathrm{eV}\right.$ [31] $)$ and $\mathrm{CoFeB}\left(\Phi_{\mathrm{CoFeB}}=4.8 \mathrm{eV}\right.$ [32]) layers. The spin accumulation generated by the Rashba effect at the $\mathrm{Zr} / \mathrm{CoFeB}$ interface is along the $-z$ direction, which is as same as the directions of $\boldsymbol{E}$ at the $\mathrm{Hf} / \mathrm{CoFeB}$ interface [24]. Therefore, the sign of the Rashba effective field is opposite to the bulk effective field from SHE.

To further confirm the $\mathrm{Zr}$-thickness-dependent sign change of the current-induced torques in the $\mathrm{Zr} / \mathrm{CoFeB} / \mathrm{MgO}$ multilayers, we performed CIMS measurements for different $\mathrm{Zr}$ thicknesses as shown in Fig. 4. We measure the Hall resistance while sweeping the in-plane pulse current. In addition to the current, a small constant in-plane magnetic field is applied along the current direction to break the symmetry, as shown in Fig. 4(a). For the sample with 2.2-nm-thick $\mathrm{Zr}$, by applying an in-plane magnetic field $H_{x}=+50 \mathrm{Oe}$, the current switches the magnetization of the $\mathrm{CoFeB}$ layer between the up and down directions, and the switching sequence is anticlockwise. By 

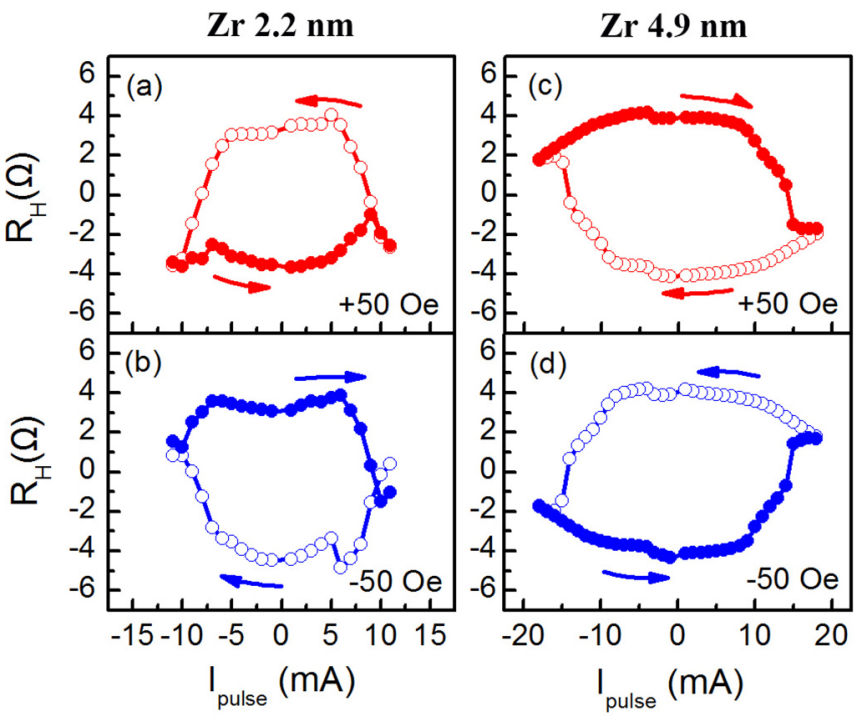

FIG. 4. Current-induced magnetization switching. $R_{H}$ as a function of current for $\operatorname{Zr}(2.2 \mathrm{~nm}) / \mathrm{CoFeB} / \mathrm{MgO}[(\mathrm{a})$, (c)] and for $\mathrm{Zr}(4.9 \mathrm{~nm}) / \mathrm{CoFeB} / \mathrm{MgO}[(\mathrm{b}),(\mathrm{d})]$. The arrows represent the current sweep direction. The width of pulsed currents is $200 \mu$ s, and the Hall resistance is measured after a $150 \mu$ s delay.

reversing the direction of the magnetic field, the switching sequence is also reversed [Fig. 4(b)]. As expected, for $t_{\mathrm{Zr}}=$ $4.9 \mathrm{~nm}$, opposite switching behavior is observed [Figs. 4(c) and 4(d)]. This confirms the Zr-thickness-dependent sign change of the torques.

Finally, we consider the OHE. The electronic band calculation of hcp $\mathrm{Zr}$ was performed using a tight-binding model based on the Slater-Koster method [33]. Detailed information on the theoretical calculation is presented in Appendix C. The lattice constants $a=0.3232 \mathrm{~nm}$ and $c=0.5147 \mathrm{~nm}$ and the Slater-Koster parameters were taken from Ref. [34]. The spin-orbit coupling was included in the Hamiltonian in the form of $\left(2 \lambda / \hbar^{2}\right) \mathbf{L} \cdot \mathbf{S}$, where $\lambda$ was set to $0.05 \mathrm{eV}$ [35]. The OHC and SHC of $\mathrm{Zr}$ were calculated by employing the Kubo formula within the linear response theory. The integration over the Brillouin zone was performed using a uniform $300 \times 300 \times 300 k$-point mesh. In Fig. 5(a), the theoretically calculated $\mathrm{OHC} \sigma_{\mathrm{OH}}$ and the $\mathrm{SHC} \sigma_{\mathrm{SH}}$ of hep $\mathrm{Zr}$ are shown as a function of the Fermi energy $E_{F}$, where $E_{F}=0$ corresponds to the actual Fermi energy. The directions of the electric field, orbital (spin) polarization, and orbital (spin) current are set to be along the $x, y$, and $z$ axes, respectively [Fig. 5(b)]. We find that $\sigma_{\mathrm{OH}}$ dominates over $\sigma_{\mathrm{SH}}$ by an order of magnitude $\left[\sigma_{\mathrm{OH}} \approx 5300(\hbar / e)(\Omega \mathrm{cm})^{-1}\right.$ and $\sigma_{\mathrm{SH}} \approx$ $-170(\hbar / e)(\Omega \mathrm{cm})^{-1}$ at $\left.E_{F}=0 \mathrm{eV}\right]$, and is about 2-3 times larger than the SHC of Pt [16]. Therefore, our experimental observation of the large current-induced torque and magnetization switching from weakly spin-orbit coupled $\mathrm{Zr}$ is likely attributed to the OHE and the orbital-to-spin conversion. If the $\mathrm{Zr}$ layer is sufficiently thick, the conversion may mostly occur in $\mathrm{Zr}$ through the SOC of $\mathrm{Zr}$ and the resulting spin Hall current will be injected to the adjacent CoFeB layer to generate a SOT. If the $\mathrm{Zr}$ layer is not sufficiently thick, the orbital Hall current may not be converted to the spin Hall current within the $\mathrm{Zr}$ layer. Then the orbital Hall current itself may be injected into
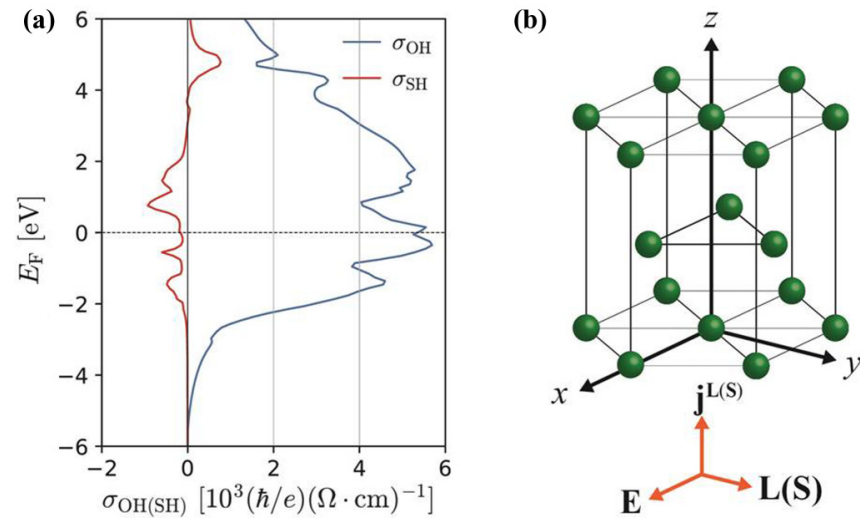

FIG. 5. Orbital and spin Hall conductivities of hep Zr. (a) $\sigma_{\mathrm{OH}}$ (blue line) and $\sigma_{\mathrm{SH}}$ (red line) of $\mathrm{Zr}$ as a function of Fermi energy $E_{F}$. See Appendix $C$ for the details of the calculations. (b) Illustration of a unit cell of hcp $\mathrm{Zr}$ and chosen coordinate axes. The orange arrows indicate the directions of the electric field, orbital (spin) polarization, and orbital (spin) current, which are set to the $x, y$, and $z$ directions, respectively.

the $\mathrm{CoFeB}$ and the conversion may mostly occur in the $\mathrm{CoFeB}$ layer instead through the SOC of CoFeB. It was recently proposed that the latter process can generate the OT [19].

Since the spin-orbit correlation is negative in $\mathrm{Zr}$ (as evidenced by the opposite signs of $\sigma_{\mathrm{OH}}$ and $\sigma_{\mathrm{SH}}$ of $\mathrm{Zr}$ ) and positive in $\mathrm{CoFeB}$, the SOT and the OT will have opposite signs and compete. The negative SOT will dominate if the $\mathrm{Zr}$ is thicker than its characteristic length $(3.2 \mathrm{~nm}$ from our experiments), and $\xi_{\text {DL }}$ will be negative as shown in Fig. 3(c) in the thick Zr regime. Furthermore, the lower bound of SHC in $\mathrm{Zr}$ can be calculated to be $\left|\sigma_{\mathrm{SH}}\right| \geqslant\left|\xi_{\mathrm{DL}} / \rho_{\mathrm{Zr}}\right| \approx$ $110(\hbar / e)(\Omega \mathrm{cm})^{-1}$, if we assume that the observed SOT originates from the SHE of $\mathrm{Zr}$ and perfect spin transparency at the $\mathrm{Zr} / \mathrm{Co}-\mathrm{Fe}-\mathrm{B}$ interface. Here, $\rho_{\mathrm{Zr}}=135 \mu \Omega \mathrm{cm}$. The estimated lower bound of SHC in $\mathrm{Zr}$ is quite close to our calculation. On the other hand, the orbital current may be injected into the adjacent $\mathrm{CoFeB}$ layer before OHE completely converts to SHE if the $\mathrm{Zr}$ is thinner than its characteristic length. Then, the positive OT becomes dominant and $\xi_{\mathrm{DL}}$ will be positive as shown in Fig. 3(c) in the thin $\mathrm{Zr}$ regime. Thus the competition between SOT and OT provides an alternative way to explain the $\mathrm{Zr}$-thickness-dependent sign change of torques.

In order to assess whether the competition between SOT and OT or the competition between SHE and the Rashba effect is more important for the sign change of torques, we have measured the interfacial Dzyaloshinskii-Moriya interaction (DMI) in our samples using current-induced AHE hysteresis loop shift measurement [13]. However, the DMI-induced effective shifts of AHE loops were within the error bars of the measurement setup, suggesting that the interfacial DMI is small. Considering that both the DMI and the Rashba-induced torque are of interfacial origin, the small DMI implies that the Rashba-induced torque is likely to be small.

The nonmagnetic metal (NM) thickness dependent sign change of the torque has been reported for $\mathrm{NM} / \mathrm{CoFeB} / \mathrm{MgO}$ for three choices of $\mathrm{NM}=\mathrm{Zr}$ (this work at $\sim 3 \mathrm{~nm}$ ), Ta [4] (at $\sim 0.5 \mathrm{~nm}$ ), and Hf [24] $(\sim 2 \mathrm{~nm})$. For $\mathrm{NM}=\mathrm{Ta}$ and $\mathrm{Hf}$, the sign change has been attributed to the competition between 
SHE and Rashba effect, and it was suggested that the signchanging thickess increases as the shunting effect becomes severe [24]. In this respect, $\mathrm{Zr}$ is abnormal since the sign change occurs at $\sim 3 \mathrm{~nm}$ (larger than the sign-changing thicknesses in Ta and Hf) despite the weaker shunting effect (the resistivity of $\mathrm{Zr} \sim 135 \mu \Omega \mathrm{cm}$ (see Appendix B) is smaller than those of Ta $(\sim 190 \mu \Omega \mathrm{cm})[4]$ and $\mathrm{Hf}(\sim 380 \mu \Omega \mathrm{cm})$ [24]). This raises the possibility that the Rashba effect may not be important for the sign change in $\mathrm{Zr}$. However further studies are necessary to clarify the main origin of the sign change unambiguously.

\section{CONCLUSIONS}

To conclude, current-induced torque effective fields exhibit a significant dependence on the $\mathrm{Zr}$ layer thickness, indicating that there are several competing effects, the Rashba effect, SHE, and OHE. Our experimental data suggest that the $\mathrm{Zr}$ thickness dependence of the sign change in torque effective fields may be influenced by the intrinsic OHE and SHE in $\mathrm{Zr}$. The $\xi_{\mathrm{DL}}$ may be further enhanced by engineering the interface between the FM and the LM and injecting more orbital current.

\section{ACKNOWLEDGMENTS}

This work was supported by National Natural Science Foundation of China (Grants No. 11574375, No. 61574169, and No. 51871018), Beijing Laboratory of Metallic Materials and Processing for Modern Transportation, Beijing Natural Science Foundation (Grant No. Z180014), and Beijing Outstanding Young Scientists Projects (Grant No. BJJWZYJH01201910005018). D.J., D.G., and H.-W.L. were supported by the Samsung Science \& Technology Foundation (Grant No. BA-1501-07). The PNR was performed at the China Spallation Neutron Source.

\section{APPENDIX A: CURRENT-INDUCED EFFECTIVE FIELD}

Low-frequency harmonic Hall voltage measurement was proposed by Pi et al. [36] and then was developed by [25]. In Ref. [25], the validity of harmonic Hall voltage measurement was tested by numerical calculations based on a macrospin model. In brief, when a current, $I$, is passed to the Hall device, the related Hall voltage, $V_{x y}=R_{x y} I$, typically contains contributions from the anomalous Hall effect (AHE) and the planar Hall effect (PHE). If we define $\Delta R_{\mathrm{AHE}}$ and $\Delta R_{\mathrm{PHE}}$ as the change in the Hall resistance due to the AHE and PHE, respectively, the Hall resistance $R_{x y}$ is expressed as [25]

$$
R_{x y}=\frac{1}{2} \Delta R_{\mathrm{AHE}} \cos \theta+\frac{1}{2} \Delta R_{\mathrm{PHE}} \sin ^{2} \theta \sin 2 \varphi,
$$

where $\theta$ and $\varphi$ are the polar and azimuthal angles, respectively, of the magnetization $M_{s}$. When a sinusoidal current is applied, the current-induced effective field oscillates in sync with the sinusoidal current. The second-harmonic Hall voltage $V_{2 \omega}$ contains information on $\Delta H_{x, y, z}$ through $\Delta \theta$ and $\Delta \varphi$. Meanwhile, the current-induced effective field $\Delta H_{x, y, z}$ can modify the magnetization angle, $\Delta \theta$ and $\Delta \varphi$, of the Hall
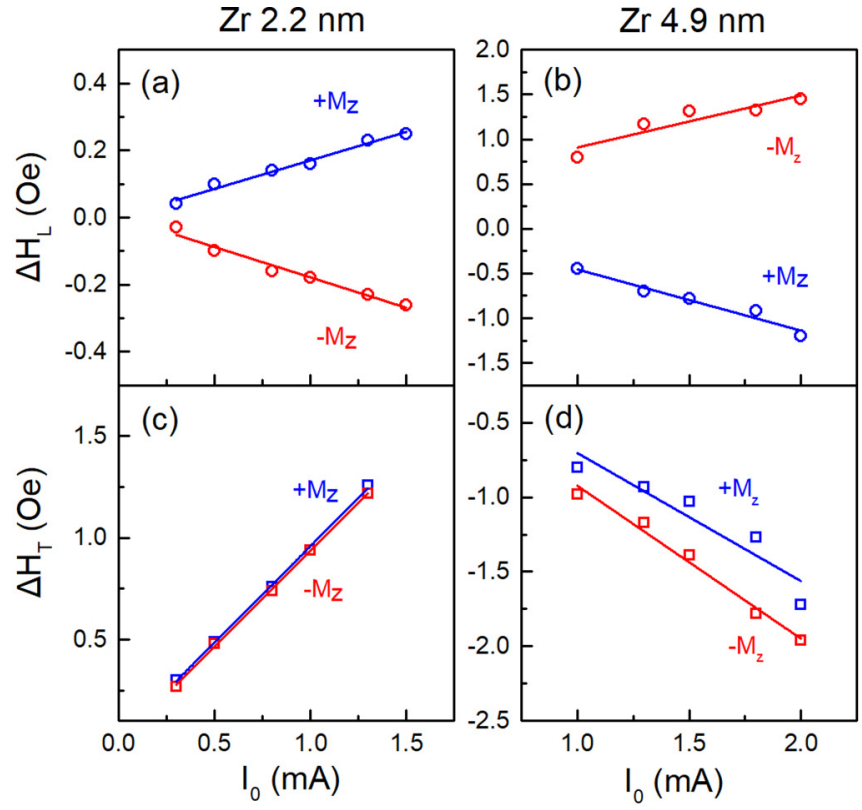

FIG. 6. Damping-like effective field $\Delta H_{L}$ and field-like effective field $\Delta H_{T}$ as a function of $I_{0}$ for $\operatorname{Zr}(2.2) / \mathrm{CoFeB} / \mathrm{MgO}[(\mathrm{a})$, (c) $]$ and $\mathrm{Zr}(4.9) / \mathrm{CoFeB} / \mathrm{MgO}$ [(b), (d)], respectively. The blue and red symbols correspond to the $+M_{z}$ and $-M_{z}$ states, respectively.

device from its equilibrium value. Thus, we can obtain

$$
\begin{aligned}
B \equiv\left(\frac{\partial V_{2 \omega}}{\partial H} / \frac{\partial^{2} V_{\omega}}{\partial H^{2}}\right)= & -\frac{1}{2}\left[\left(\Delta H_{x} \mp 2 \xi \cos 2 \varphi_{H} \Delta H_{y}\right) \cos \varphi_{H}\right. \\
& \left.+\left(\Delta H_{y} \pm 2 \xi \cos 2 \varphi_{H} \Delta H_{x}\right) \sin \varphi_{H}\right]
\end{aligned}
$$

Here, $\xi \equiv \Delta R_{\mathrm{PHE}} / \Delta R_{\mathrm{AHE}}$, which is the ratio of the PHE and AHE resistances [25]. Finally, we obtain

$$
\begin{aligned}
& \Delta H_{x}=-2 \frac{\left(B_{x} \pm 2 \xi B_{y}\right)}{1-4 \xi^{2}}, \\
& \Delta H_{y}=-2 \frac{\left(B_{y} \pm 2 \xi B_{x}\right)}{1-4 \xi^{2}} .
\end{aligned}
$$

When $\xi$ is very small-for instance, $\xi$ is about 0.03 for our perpendicularly magnetized $\mathrm{Zr} / \mathrm{CoFeB} / \mathrm{MgO}$ samplesthe above Eqs. (A3) and (A4) can be simplified as Eq. (1) that we used in the text.

As shown in Figs. 6(a)-6(d), we calculated the $\Delta H_{L}$ and $\Delta H_{T}$, which are plotted as a function of input bias current amplitude $\left(I_{0}\right)$ of the sinusoidal signal for the samples with $\mathrm{Zr}$ thicknesses of $2.2 \mathrm{~nm}$ and $4.9 \mathrm{~nm}$, respectively. It is clear that the effective fields vary linearly with $I_{0}$, indicating that the effects of Joule heating are negligible in the measured $I_{0}$ range [23]. Similarly to the previous reports [4,24], only the sign of the trend for $\Delta H_{L}$ with the bias current amplitude is opposite for the different magnetization states. Notice that the sign of the trend for both effective fields is inverse for the samples with $\mathrm{Zr}$ thicknesses of 2.2 and $4.9 \mathrm{~nm}$, which indicates the different dominating mechanisms in the currentinduced torques. 


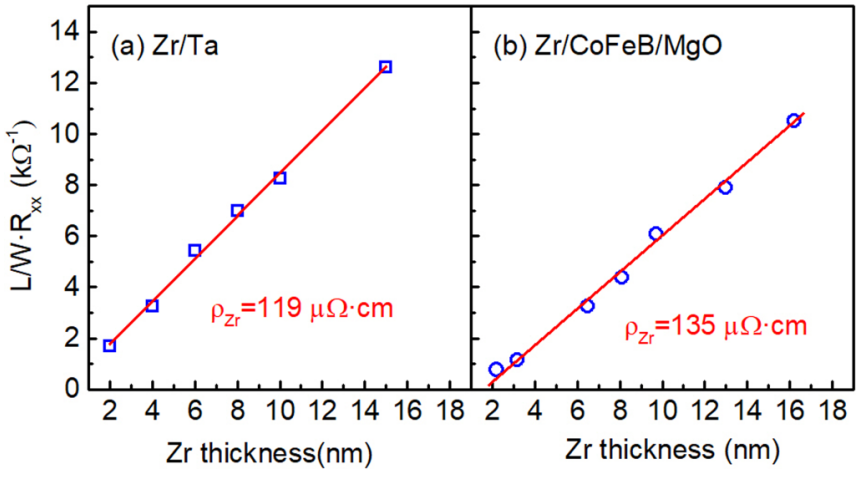

FIG. 7. $L /\left(R_{x x} W\right)$ plotted as a function of the $\mathrm{Zr}$ thickness for the $\mathrm{Zr} / \mathrm{Ta}$ bilayer (a) and the $\mathrm{Zr} / \mathrm{CoFeB} / \mathrm{MgO}$ multilayer (b). The lines indicate a linear fit to the data.

\section{APPENDIX B: THE RESISTIVITY OF Zr}

Figure 7 shows the plot of normalized inverse channel resistance, $L /\left(R_{x x} W\right)$, as a function of $t_{\mathrm{Zr}}$, where $L$ and $W$ are the length and width of the Hall bar channel, respectively, and $R_{x x}$ is the longitudinal channel resistance. From the fittings, we obtain $\rho_{\mathrm{Zr}}=119 \mu \Omega \mathrm{cm}$ in the $\mathrm{Zr} / \mathrm{Ta}$ bilayer, while $\rho_{\mathrm{Zr}}=$ $135 \mu \Omega \mathrm{cm}$ in the $\mathrm{Zr} / \mathrm{CoFeB} / \mathrm{MgO}$ multilayer. The increase of $\mathrm{Zr}$ resistivity in the $\mathrm{Zr} / \mathrm{CoFeB} / \mathrm{MgO}$ multilayer may be due to the $\mathrm{B}$ diffusion in the $\mathrm{Zr}$, which is consistent with the PNR results in Fig. 1. To further account for the currentshunting effect, the current density in $\mathrm{Zr}$ is calculated by $J_{e}=$ $J_{0}\left[t_{\mathrm{CoFeB}} \rho_{\mathrm{Zr}} /\left(t_{\mathrm{CoFeB}} \rho_{\mathrm{Zr}}+t_{\mathrm{Zr}} \rho_{\mathrm{CoFeB}}\right)\right]$, where $J_{0}=I_{0} /\left(W t_{\mathrm{Zr}}\right)$ is the nominal current density in the $\mathrm{Zr}$ layer. $t_{\mathrm{Zr}}$ and $t_{\mathrm{CoFeB}}$ are the thickness of $\mathrm{Zr}$ and $\mathrm{CoFeB}$, respectively, and $\rho_{\mathrm{Zr}}$ and $\rho_{\mathrm{CoFeB}}$ are the resistivities of $\mathrm{Zr}$ and $\mathrm{CoFeB}$, respectively. The resistivity of the individual layer was determined by channel resistance of the Hall bar verse thin film thickness measurements, which gives $\rho_{\mathrm{CoFeB}}=76 \mu \Omega \mathrm{cm}$ and $\rho_{\mathrm{Zr}}=$ $135 \mu \Omega \mathrm{cm}$. Both the decrease of resistivity $\mathrm{CoFeB}$ and the increase of its magnetization may also be due to the B diffusion.

\section{APPENDIX C: THEORETICAL CALCULATIONS OF ORBITAL AND SPIN HALL CONDUCTANCE}

In order to obtain the energy eigenvalues and corresponding eigenstates of hcp Zr, we employed the tightbinding method based on the Slater-Koster method [33]. We introduced 36 orthogonal atomic-orbital basis sets $\left\{\left|\phi_{n \sigma \mathbf{R}}\right\rangle\right\}$ for each Bravais lattice vector $\mathbf{R}$ with orbital $n=$ $s, p_{x}, p_{y}, p_{z}, d_{z^{2}}, d_{x^{2}-y^{2}}, d_{x y}, d_{y z}, d_{z x}$ for two atomic sites in a unit cell, and two spins $\sigma=\uparrow, \downarrow$. Then the $36 \times 36$ Hamiltonian $H(\mathbf{R})$ matrix for each $\mathbf{R}$ is written in terms of the Slater-Koster parameters taken from Ref. [34]. The spin-orbit coupling for the $l=s, p, d$ orbital was included in the form of $\left(2 \lambda_{l} / \hbar^{2}\right) \mathbf{L}^{(l)} \cdot \mathbf{S}$, where $\lambda_{l}$ is the spin-orbit

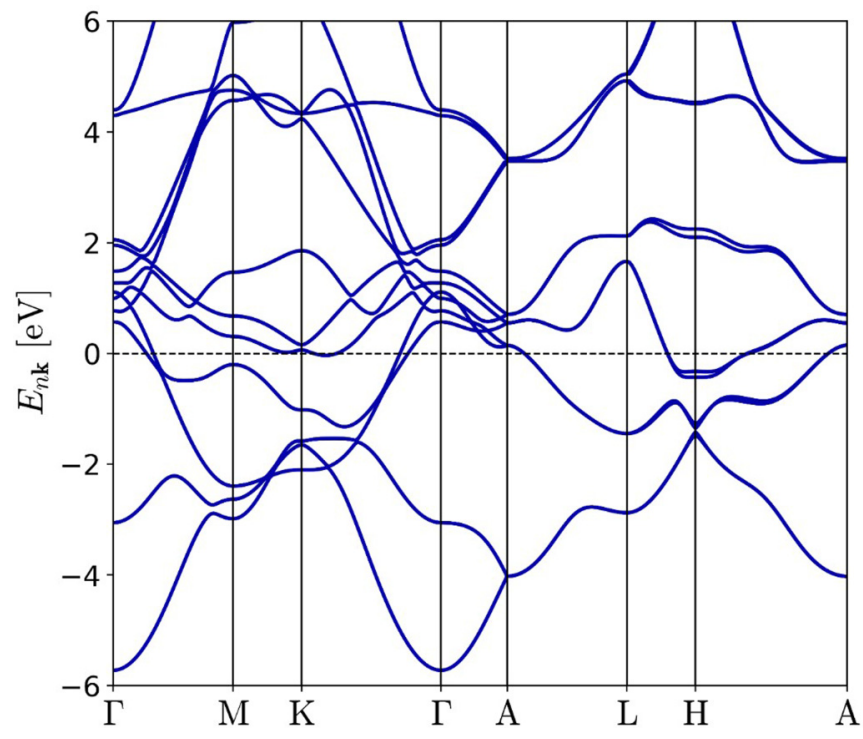

FIG. 8. Band structure of hep $\mathrm{Zr}$ obtained from tight-binding calculations.

coupling constant, and $\mathbf{S}$ and $\mathbf{L}^{(l)}$ are the spin and orbital angular momentum operators for the $l$ orbital, respectively. Here $\mathbf{L}^{(l)}$ is defined with respect to the center of each atom. We set $\lambda_{l}$ to be a spin-orbit coupling constant for the outermost electron $0.05 \mathrm{eV}$ [35], which is assumed to be independent of $l$. The $k$-space representation of the Hamiltonian $H(\mathbf{k})$ is obtained from a basis set of Bloch-like states $\left|\psi_{n \sigma \mathbf{k}}\right\rangle=$ $(1 / \sqrt{N}) \sum_{\mathbf{R}} e^{i \mathbf{k} \cdot \mathbf{R}}\left|\phi_{n \sigma \mathbf{R}}\right\rangle$. Solving an eigenvalue equation for $H(\mathbf{k})$ gives the electronic band structure of hcp $\mathrm{Zr}$ as shown in Fig. 8.

The calculations of $\mathrm{OHC}$ and SHC were carried out using the Kubo formula,

$$
\begin{aligned}
\sigma_{\mathrm{OH}(\mathrm{SH})}= & -e \hbar \sum_{n, m} \int \frac{d^{3} \mathbf{k}}{(2 \pi)^{3}}\left(f_{n \mathbf{k}}-f_{m \mathbf{k}}\right) \\
& \times \operatorname{Im}\left[\frac{\left\langle u_{n \mathbf{k}}\left|j_{z}^{X_{y}}(\mathbf{k})\right| u_{m \mathbf{k}}\right\rangle\left\langle u_{m \mathbf{k}}\left|v_{x}(\mathbf{k})\right| u_{n \mathbf{k}}\right\rangle}{\left(E_{n \mathbf{k}}-E_{m \mathbf{k}}+i \eta\right)^{2}}\right],
\end{aligned}
$$

where $e>0$ is the elementary charge, $\hbar$ is the reduced Planck constant, $f_{n \mathbf{k}}$ is the Fermi-Dirac distribution function for room temperature, $\left|u_{n \mathbf{k}}\right\rangle$ is a periodic part of the Bloch state with energy eigenvalue $E_{n \mathbf{k}}, v_{x}(\mathbf{k})=\partial_{k_{x}} H(\mathbf{k}) / \hbar$ is the $x$ component of the velocity operator, and $\eta$ was set to $25 \mathrm{meV}$. Here $j_{z}^{X_{y}}(\mathbf{k})$ is the $z$ component of the orbital (spin) current operator carrying $y$-directional orbital (spin) angular momentum,

$$
j_{z}^{X_{y}}(\mathbf{k})=\frac{1}{2}\left[X_{y} v_{z}(\mathbf{k})+v_{z}(\mathbf{k}) X_{y}\right]
$$

with $X_{y}=L_{y}\left(S_{y}\right)$. The $k$ integration over the Brillouin zone in Eq. (C1) was performed on a uniformly distributed $300 \times$ $300 \times 300 k$-point mesh.
[1] A. Brataas, A. D. Kent, and H. Ohno, Current-induced torques in magnetic materials, Nat. Mater. 11, 372 (2012).
[2] I. M. Miron, K. Garello, G. Gaudin, P. J. Zermatten, M. V. Costache, S. Auffret, S. Bandiera, B. Rodmacq, A. Schuhl, 
and P. Gambardella, Perpendicular switching of a single ferromagnetic layer induced by in-plane current injection, Nature (London) 476, 189 (2011).

[3] L. Liu, C.-F. Pai, Y. Li, H. Tseng, D. Ralph, and R. Buhrman, Spin-torque switching with the giant spin Hall effect of tantalum, Science 336, 555 (2012).

[4] J. Kim, J. Sinha, M. Hayashi, M. Yamanouchi, S. Fukami, T. Suzuki, S. Mitani, and H. Ohno, Layer thickness dependence of the current-induced effective field vector in $\mathrm{Ta}|\mathrm{CoFeB}| \mathrm{MgO}$, Nat. Mater. 12, 240 (2013).

[5] K. Garello, I. M. Miron, C. O. Avci, F. Freimuth, Y. Mokrousov, S. Blugel, S. Auffret, O. Boulle, G. Gaudin, and P. Gambardella, Symmetry and magnitude of spin-orbit torques in ferromagnetic heterostructures, Nat. Nanotechnol. 8, 587 (2013).

[6] S. Emori, U. Bauer, S. M. Ahn, E. Martinez, and G. S. Beach, Current-driven dynamics of chiral ferromagnetic domain walls, Nat. Mater. 12, 611 (2013).

[7] G. Yu, P. Upadhyaya, Y. Fan, J. G. Alzate, W. Jiang, K. L. Wong, S. Takei, S. A. Bender, L. T. Chang, Y. Jiang et al., Switching of perpendicular magnetization by spin-orbit torques in the absence of external magnetic fields, Nat. Nanotechnol. 9, 548 (2014)

[8] X. Qiu, K. Narayanapillai, Y. Wu, P. Deorani, D. H. Yang, W. S. Noh, J. H. Park, K. J. Lee, H. W. Lee, and H. Yang, Spin-orbittorque engineering via oxygen manipulation, Nat. Nanotechnol. 10, 333 (2015).

[9] S. Fukami, C. Zhang, S. DuttaGupta, A. Kurenkov, and H. Ohno, Magnetization switching by spin-orbit torque in an antiferromagnet-ferromagnet bilayer system, Nat. Mater. 15, 535 (2016)

[10] A. van den Brink, G. Vermijs, A. Solignac, J. Koo, J. T. Kohlhepp, H. J. M. Swagten, and B. Koopmans, Field-free magnetization reversal by spin-Hall effect and exchange bias, Nat. Commun. 7, 10854 (2016).

[11] L. Liu, O. J. Lee, T. J. Gudmundsen, D. C. Ralph, and R. A. Buhrman, Current-Induced Switching of Perpendicularly Magnetized Magnetic Layers Using Spin Torque from the Spin Hall Effect, Phys. Rev. Lett. 109, 096602 (2012).

[12] C.-F. Pai, L. Liu, Y. Li, H. W. Tseng, D. C. Ralph, and R. A. Buhrman, Spin transfer torque devices utilizing the giant spin Hall effect of tungsten, Appl. Phys. Lett. 101, 122404 (2012).

[13] T.-Y. Chen, H.-I. Chan, W.-B. Liao, and C.-F. Pai, CurrentInduced Spin-Orbit Torque and Field-Free Switching in MoBased Magnetic Heterostructures, Phys. Rev. Appl. 10, 044038 (2018).

[14] T. C. Chuang, C. F. Pai, and S. Y. Huang, Cr-Induced Perpendicular Magnetic Anisotropy and Field-Free Spin-Orbit-Torque Switching, Phys. Rev. Appl. 11, 061005 (2019).

[15] T. Tanaka, H. Kontani, M. Naito, T. Naito, D. S. Hirashima, K. Yamada, and J. Inoue, Intrinsic spin Hall effect and orbital Hall effect in $4 d$ and $5 d$ transition metals, Phys. Rev. B 77, 165117 (2008).

[16] H. Kontani, T. Tanaka, D. S. Hirashima, K. Yamada, and J. Inoue, Giant Orbital Hall Effect in Transition Metals: Origin of Large Spin and Anomalous Hall Effects, Phys. Rev. Lett. 102, 016601 (2009).

[17] D. Go, D. Jo, C. Kim, and H. W. Lee, Intrinsic Spin and Orbital Hall Effects from Orbital Texture, Phys. Rev. Lett. 121, 086602 (2018).
[18] D. Jo, D. Go, and H.-W. Lee, Gigantic intrinsic orbital Hall effects in weakly spin-orbit coupled metals, Phys. Rev. B 98, 214405 (2018)

[19] D. Go and H.-W. Lee, Orbital torque: Torque generation by orbital current injection, arXiv:1903.01085.

[20] T. Zhu, F. F. Chang, and X. Z. Zhan, Interface induced enhancement of inverse spin Hall voltage in $\mathrm{NiFe} / \mathrm{Pt}$ bilayers capped by MgO layer, J. Phys. Condens. Matter 31, 285801 (2019).

[21] SimulReflec, Leon Brillouin CEA/CNRS UMR12, Copyright (C) Lab, 2011, free software available at http://www-llb.cea.fr/ prism/programs/simulreflec/simulreflec.html.

[22] T. Zhu, Y. Yang, R. C. Yu, H. Ambaye, V. Lauter, and J. Q. $\mathrm{Xiao}$, The study of perpendicular magnetic anisotropy in $\mathrm{CoFeB}$ sandwiched by $\mathrm{MgO}$ and tantalum layers using polarized neutron reflectometry, Appl. Phys. Lett. 100, 202406 (2012).

[23] K. Ueda, C.-F. Pai, A. J. Tan, M. Mann, and G. S. D. Beach, Effect of rare earth metal on the spin-orbit torque in magnetic heterostructures, Appl. Phys. Lett. 108, 232405 (2016).

[24] R. Ramaswamy, X. Qiu, T. Dutta, S. D. Pollard, and $\mathrm{H}$. Yang, Hf thickness dependence of spin-orbit torques in $\mathrm{Hf} / \mathrm{CoFeB} / \mathrm{MgO}$ heterostructures, Appl. Phys. Lett. 108, 202406 (2016).

[25] M. Hayashi, J. Kim, M. Yamanouchi, and H. Ohno, Quantitative characterization of the spin-orbit torque using harmonic Hall voltage measurements, Phys. Rev. B 89, 144425 (2014).

[26] X. Wang and A. Manchon, Diffusive Spin Dynamics in Ferromagnetic Thin Films with a Rashba Interaction, Phys. Rev. Lett. 108, 117201 (2012).

[27] K.-W. Kim, S.-M. Seo, J. Ryu, K.-J. Lee, and H.-W. Lee, Magnetization dynamics induced by in-plane currents in ultrathin magnetic nanostructures with Rashba spin-orbit coupling, Phys. Rev. B 85, 180404 (2012).

[28] D. Pesin and A. H. MacDonald, Quantum kinetic theory of current-induced torques in Rashba ferromagnets, Phys. Rev. B 86, 014416 (2012)

[29] H. Kurebayashi, J. Sinova, D. Fang, A. Irvine, T. Skinner, J. Wunderlich, V. Novák, R. Campion, B. Gallagher, and E. Vehstedt, An antidamping spin-orbit torque originating from the Berry curvature, Nat. Nanotechnol. 9, 211 (2014).

[30] S. Zhang, Spin Hall Effect in the Presence of Spin Diffusion, Phys. Rev. Lett. 85, 393 (2000).

[31] H. B. Michaelson, The work function of the elements and its periodicity, J. Appl. Phys. 48, 4729 (1977).

[32] T. Uhrmann, T. Dimopoulos, H. Brückl, V. K. Lazarov, A. Kohn, U. Paschen, S. Weyers, L. Bär, and M. Rührig, Characterization of embedded $\mathrm{MgO} /$ ferromagnet contacts for spin injection in silicon, J. Appl. Phys. 103, 063709 (2008).

[33] J. C. Slater and G. F. Koster, Simplified LCAO method for the periodic potential problem, Phys. Rev. 94, 1498 (1954).

[34] Handbook of the Band Structure of Elemental Solids, 2nd ed., edited by D. A. Papaconstantopoulos (Springer, New York, 2015), p. 199.

[35] K. Shanavas, Z. S. Popović, and S. Satpathy, Theoretical model for Rashba spin-orbit interaction in $d$ electrons, Phys. Rev. B 90, 165108 (2014).

[36] U. H. Pi, K. W. Kim, J. Y. Bae, S. C. Lee, Y. J. Cho, K. S. Kim, and S. Seo, Tilting of the spin orientation induced by Rashba effect in ferromagnetic metal layer, Appl. Phys. Lett. 97, 162507 (2010) 